Revue de droit comparé du travail et de la sécurité sociale

3 | 2017

Le travail dans l'économie informelle, un défi pour le droit social

\title{
La régulation de l'utilisation des TIC sur le lieu de travail
}

Gintarè Tamašauskaitè-Janickè

\section{OpenEdition}

\section{Journals}

Édition électronique

URL : https://journals.openedition.org/rdctss/453

DOI : $10.4000 /$ rdctss. 453

ISSN : 2262-9815

Éditeur

Centre de droit comparé du travail et de la sécurité sociale

Édition imprimée

Date de publication : 1 septembre 2017

Pagination : 216-219

ISSN : $2117-4350$

Référence électronique

Gintarè Tamašauskaitè-Janickè, «La régulation de l'utilisation des TIC sur le lieu de travail », Revue de droit comparé du travail et de la sécurité sociale [En ligne], 3 | 2017, mis en ligne le 01 novembre 2017 consulté le 04 décembre 2021. URL : http://journals.openedition.org/rdctss/453 ; DOI : https://doi.org/ $10.4000 /$ rdctss. 453

\section{(c) (i) (9)}

Revue de droit comparé du travail et de la sécurité sociale est mise à disposition selon les termes de la Licence Creative Commons Attribution - Pas d'Utilisation Commerciale - Pas de Modification 4.0 International. 


\title{
GINTARE் TAMAŠAUSKAITE்-JANICKE் Université de Vilnius
}

\author{
LA RÉGULATION DE L'UTILISATION DES TIC SUR LE LIEU DE TRAVAIL
}

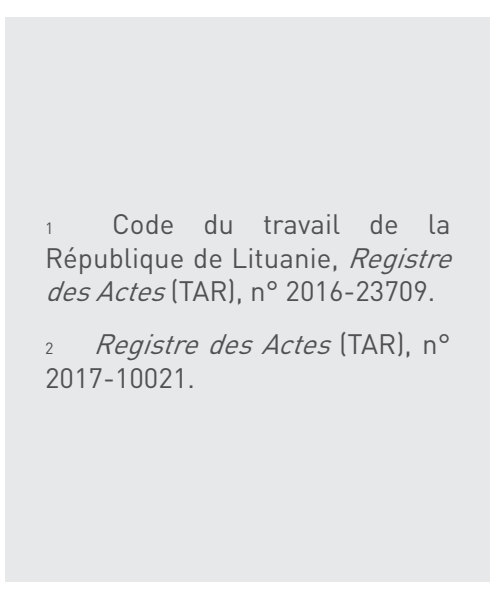

Fin 2016, à la veille des élections parlementaires, le nouveau Code du travail ${ }^{1}$ a été adopté par le Parlement lituanien (Seimas) ; il ne devait entrer en vigueur qu'à partir du 1er janvier 2017. Mais, les membres du Seimas nouvellement élus ont décidé de reporter cette entrée en vigueur au 1er juillet 2017 après l'adoption d'amendements le 6 juin $2017^{2}$.

La réforme du droit du travail lituanien est le résultat d'une approche libérale de la régulation des rapports du travail. La nouvelle règlementation transforme radicalement les règles héritées de l'époque soviétique lavec des modifications visibles et influencées tout particulièrement par l'harmonisation du droit national avec le droit de l'UE). On doit souligner que le droit du travail antérieur ne satisfaisait personne en raison de son manque total de souplesse si bien que les parties au contrat de travail cherchaient souvent des moyens de contourner ces règles. La plupart des règles de l'ancien Code de travail n'étaient pas appliquées en réalité. Par conséquent, la révision systématique et en profondeur des règles opérée avec l'entrée en vigueur du nouveau code entrainera des transformations d'envergure des rapports entre les parties à la relation de travail et leurs représentants confrontés aux défis contemporains d'une économie et d'une société du numérique.

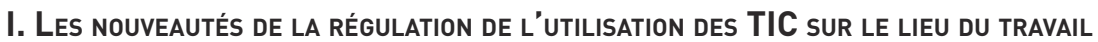

La rapidité des technologies et la mondialisation ont créé de nouveaux enjeux concernant la protection des données à caractère personnel sur le lieu du travail. Le droit du travail s'est vu attribuer des fonctions supplémentaires pour réagir aux problèmes posés par la régulation juridique de la protection de la vie privée des salariés.

Pour la première fois en droit du travail national, l'article 27 du Code de travail définit les principes fondamentaux du droit au respect de la vie privée du salarié et de la protection de ses données à caractère personnel sur le lieu du travail :

- il prévoit impérativement l’obligation de l'employeur de respecter le droit à la vie privée du salarié et d'assurer la protection de ses données personnelles;

- il interdit à l'employeur de traiter les données à caractère personnel du salarié non liées aux besoins du travail ou de transférer ces données à des tiers, sauf exceptions prévues par d'autres lois ;

- il préconise à l'employeur d'adopter une norme d'entreprise sur l'utilisation des TIC, sur la surveillance et le contrôle des salariés sur le lieu du travail ainsi que d'en informer les salarié(e)s; 
- il impose à l'entreprise ayant en moyenne plus de cinquante salariés, d'adopter une politique locale de stockage des données à caractère personnel des travailleurs, des mesures de mise en œuvre et d'en informer les salariélels de manière régulière ;

- il établit que le secret des communications personnelles du salarié(e) ne peut pas être violé par l'employeur même lorsqu'il utilise son droit de propriété ou de gestion des TIC sur le lieu du travail ;

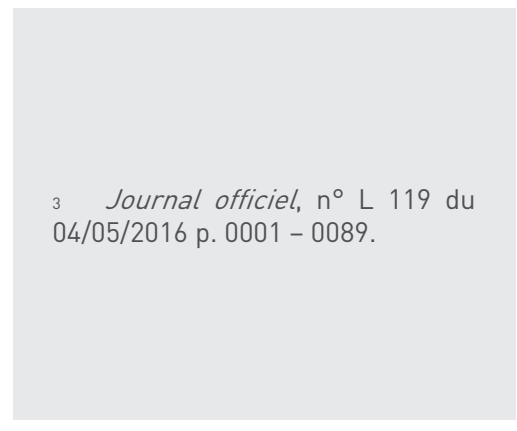

- il dispose que la surveillance vidéo et l'enregistrement audio sur le lieu du travail peuvent être utilisés lorsque les spécificités du travail supposent d'assurer la sécurité des personnes, des biens ou de l'ordre public et dans le cas où d'autres moyens ou mesures sont inadéquats et/ou inappropriés aux fins indiquées; mais la surveillance ne peut pas être utilisée directement pour contrôler la qualité et la quantité de travail. Il est nécessaire d'informer les salariés de la surveillance vidéo et l'enregistrement audio en utilisant les lieux d'affichage et d'information dans les locaux sous surveillance ;

- La protection juridique des données à caractère personnel des salariés et de l'application du droit au respect de la vie privée du salarié peuvent être établies et régulées par d'autres lois et normes de droit du travail.

\section{La RÉgulation de L'Utilisation des TIC SUR le LIEU dU tRAVAIL DANS LE CONTEXTE dE LA RÉforme du DROIT DE L'UE SUR LA PROTECTION DES DONNÉES PERSONNELLES}

Le changement de la régulation de la protection des données personnelles au niveau de l'UE par le règlement 2016/679 du 27 avril 2016 relatif à la protection des personnes physiques à l'égard du traitement des données à caractère personnel et à la libre circulation de ces données, abrogeant la directive 95/46/CE (ci-après - «RGPD »)3 et cherchant un compromis entre les intérêts des salariés et des employeurs, sera applicable aux entreprises privées et publiques à partir du 25 mai 2018.

Le RGPD prévoit également des règles relatives aux traitements effectués dans le cadre des relations de travail. En principe, on peut les classer de la manière suivante :

(1) les règles attribuant une légitimité au traitement des données dans le contexte des relations de travail ;

(2) la lex specialis déterminant des clauses et une régulation spécifiques uniquement dans le domaine des relations de travail. 
A. Les articles 13 et 75 du préambule du RGPD établissent la légitimité du traitement de données à caractère personnel dans le contexte des relations de travail. Le RGPD repose sur l'objectif d'assurer un niveau cohérent de protection des personnes physiques dans l'ensemble de l'UE, d'éviter que des divergences n'entravent la libre circulation des données personnelles au sein du marché intérieur, et de garantir la sécurité juridique et la transparence aux opérateurs économiques, y compris les micro, petites et moyennes entreprises, pour offrir aux personnes physiques de tous les États membres un même niveau de droits opposables, d'obligations et de responsabilités pour les pesonnes en charge du traitement et les sous-traitants, d'assurer une surveillance cohérente du traitement des données à caractère personnel et d'assurer des sanctions équivalentes dans tous les États membres. Le RGPD convient qu'il est nécessaire que la libre circulation des données à caractère personnel au sein de l'UE ne soit ni limitée ni interdite pour des motifs liés à la protection des personnes physiques à l'égard du traitement des données personnelles afin que le marché intérieur fonctionne correctement.

En plus, des risques pour les droits et libertés des personnes physiques, dont le degré de probabilité et de gravité varie, des dommages physiques, matériels ou moraux peuvent résulter du traitement de données à caractère personnel. Toutefois, il est essentiel de tenir compte de la situation particulière des petites et moyennes entreprises en ce qui concerne l'application de la nouvelle régulation.

B. Le RGPD déclare que le droit des États membres ou des conventions collectives, y compris des « accords d'entreprise » peuvent prévoir des règles spécifiques relatives au traitement des données à caractère personnel des salariés dans le cadre des relations de travail, notamment les conditions dans lesquelles les données à caractère personnel dans ce cadre peuvent être traitées sur la base du consentement du salarié, aux fins de recrutement, de l'exécution du contrat de travail, dans le respect des obligations fixées par la loi ou par des conventions collectives, de la gestion, de la planification et de l'organisation du travail, de l'égalité et de la diversité sur le lieu du travail, de la santé et de la sécurité au travail, et dans l'exercice et la jouissance des droits et des avantages liés à l'emploi, individuellement ou collectivement, ainsi qu'aux fins de la résiliation de la relation de travail.

L'article 9 du RGPD prévoit l'interdiction du traitement de données à caractère personnel révélant l'origine raciale ou ethnique, les opinions politiques, les convictions religieuses ou philosophiques, l'appartenance syndicale, ainsi que le traitement des données génétiques, des données biométriques aux fins d'identifier une personne physique de manière unique, des données concernant la santé ou des données concernant la vie sexuelle ou l'orientation sexuelle. Néanmoins, les deuxièmes $b$ et $\mathrm{h}$ alinéas de cet article prévoient deux exceptions : quand (1) le traitement est nécessaire aux fins de l'exécution des obligations et de l'exercice des droits propres au responsable du traitement ou à la personne concernée en matière de droit du travail, de la sécurité sociale et de la protection sociale, dans la mesure où ce traitement est autorisé par le droit de l'UE, par le droit d'un État membre ou par une convention collective conclue en vertu du droit d'un État membre qui prévoit des garanties appropriées pour les droits fondamentaux et les intérêts de la personne concernée ; et 
quand (2) le traitement est nécessaire aux fins de médecine préventive ou de médecine du travail, de l'appréciation de la capacité de travail du travailleur, de diagnostics médicaux, de prise en charge sanitaire ou sociale, ou de gestion des systèmes et des services de soins de santé ou de protection sociale sur la base du droit de l'UE, du droit d'un État membre ou en vertu d'un contrat conclu avec un professionnel de la santé.

En conséquence et eu égard à la compétence limitée d'adopter des normes pour réguler des relations de travail par le droit du l'UE, l'article 88 du RGPD reconnaît le droit pour les États membres de prévoir, par la loi ou au moyen de conventions collectives, des règles plus spécifiques pour assurer la protection des droits et libertés en ce qui concerne le traitement des données à caractère personnel des salariés dans le cadre des relations de travail, pour ce qui est :

- du recrutement ;

- de l'exécution du contrat de travail, y compris le respect des obligations fixées par la loi ou par des conventions collectives ;

- de la gestion ;

- de la planification et de l'organisation du travail ;

- de l'égalité et de la diversité sur le lieu du travail ;

- de la santé et de la sécurité au travail ;

- de la protection des biens appartenant à l'employeur ou au client ;

- de l'exercice et de la jouissance des droits et des avantages liés à l'emploi, individuellement ou collectivement :

- de la résiliation de la relation de travail.

Ces règles nationales comprennent des mesures appropriées et spécifiques pour protéger la dignité humaine, les intérêts légitimes et les droits fondamentaux des personnes concernées, et accordent une attention particulière à la transparence du traitement, au transfert de données à caractère personnel au sein d'un groupe d'entreprises, ou d'un groupe d'entreprises engagées dans une activité économique conjointe et aux systèmes de contrôle sur le lieu du travail.

En conclusion, en Lituanie, avant l'adoption des normes sur l'utilisation de TIC par le droit du travail seules des normes générales relatives à la protection de données à caractère personnel et au droit au respect de la vie privée du salarié étaient appliquées dans le domaine de l'emploi. Les interdictions et les obligations concrètes renforceront la protection des droits des salariés spécifiques et vulnérables tout en établissant un compromis entre les intérêts des travailleurs et des employeurs. Les nouvelles dispositions du Code du travail correspondent au RGPD et aux idées développées par la réforme de la protection des données à caractère personnel au niveau de l'UE. 